\title{
Cell Adhesion on RGD-Displaying Knottins with Varying Numbers of Tryptophan Amino Acids to Tune the Affinity for Assembly on Cucurbit[8]uril Surfaces
}

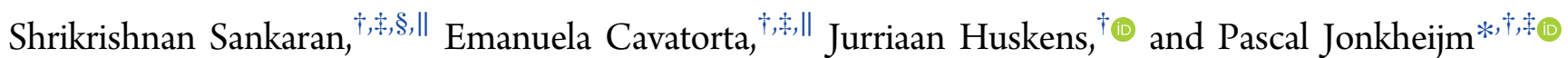

${ }^{\dagger}$ Molecular Nanofabrication Group, MESA+ Institute for Nanotechnology, Department of Science and Technology and ${ }^{\ddagger}$ Bioinspired Molecular Engineering Laboratory, MIRA Institute for Biomedical Technology and Technical Medicine and Molecular Nanofabrication Group, MESA+ Institute for Nanotechnology, Department of Science and Technology, University of Twente, 7500 AE Enschede, The Netherlands

Supporting Information

ABSTRACT: Cell adhesion is studied on multivalent knottins, displaying RGD ligands with a high affinity for integrin receptors, that are assembled on $\mathrm{CB}[8]$-methylviologenmodified surfaces. The multivalency in the knottins stems from the number of tryptophan amino acid moieties, between 0 and 4, that can form a heteroternary complex with cucurbit [8]uril $(\mathrm{CB}[8])$ and surface-tethered methylviologen $\left(\mathrm{MV}^{2+}\right)$. The binding affinity of the knottins with $\mathrm{CB}[8]$ and $\mathrm{MV}^{2+}$ surfaces was evaluated using surface plasmon resonance spectroscopy. Specific binding occurred, and the affinity increased with the valency of tryptophans on the knottin. Additionally, increased multilayer formation was observed, attributed to homoternary

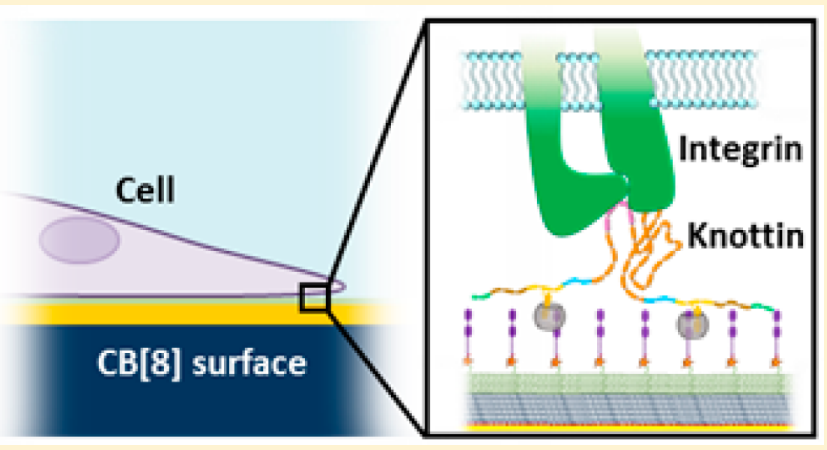
complex formation between tryptophan residues of different knottins and $\mathrm{CB}[8]$. Thus, we were able to control the surface coverage of the knottins by valency and concentration. Cell experiments with mouse myoblast $(\mathrm{C} 2 \mathrm{C} 12)$ cells on the self-assembled knottin surfaces showed specific integrin recognition by the RGD-displaying knottins. Moreover, cells were observed to elongate more on the supramolecular knottin surfaces with a higher valency, and in addition, more pronounced focal adhesion formation was observed on the higher-valency knottin surfaces. We attribute this effect to the enhanced coverage and the enhanced affinity of the knottins in their interaction with the CB[8] surface. Collectively, these results are promising for the development of biomaterials including knottins via $\mathrm{CB}[8]$ ternary complexes for tunable interactions with cells.

\section{INTRODUCTION}

Cells are surrounded by a highly dynamic extracellular matrix (ECM) and respond to various factors in the ECM environment, among which chemical and mechanical cues play a major role. ${ }^{1,2}$ Such cues can initiate a cascade of instructions for adhesion, spreading, proliferation, migration, and differentiation and eventually even drive the formation and the development of tissues. Among the cell surface receptors that signal these cues, members of the integrin family recruit multiple proteins that eventually form so-called focal adhesions between the cell's cytoskeleton and the ECM. ${ }^{3,4}$ A synthetic Arg-Gly-Asp (RGD) peptide motif, present on several ECM proteins such as fibronectin or vitronectin, has been identified to selectively bind to integrin receptors. ${ }^{5}$ The development of biomimetic materials incorporating this short RGD peptide sequence has resulted in much progress in understanding cell responses on biomaterials that mimic ECM cues. ${ }^{6,7}$ However, the introduction of dynamic and stimuli-responsive molecular constituents in such biomimetic materials has only recently gained attention. $^{8-10}$
Supramolecular chemistry offers a powerful approach to designing systems in which ligands engage with their cell receptors in a dynamic, reversible, and stimulus-sensitive manner. ${ }^{11}$ Biomimetic matrices have been reported on the basis of host-guest chemistry using cyclodextrins and cucurbit[n] urils for the tunable, modular, and responsive presentation of RGD peptide ligands for cell adhesion. ${ }^{11-19}$ For example, we have reported the development of a self-assembled monolayer (SAM) for the dynamic display of linear RGD sequences through the formation of a $\mathrm{CB}[8]$ heteroternary complex between surface-bound methyl viologen $\left(\mathrm{MV}^{2+}\right)$ and naphtholmodified RGD. ${ }^{12,20}$ On these surfaces, it was also possible to locally release ligands through electrochemical stimuli with subcellular resolution. ${ }^{12}$ Recent FluidFM measurements revealed that the rupture forces of cells adhered to interfaces

Special Issue: Surfaces and Interfaces for Molecular Monitoring

Received: March 1, 2017

Revised: $\quad$ May 8, 2017

Published: May 18, 2017 


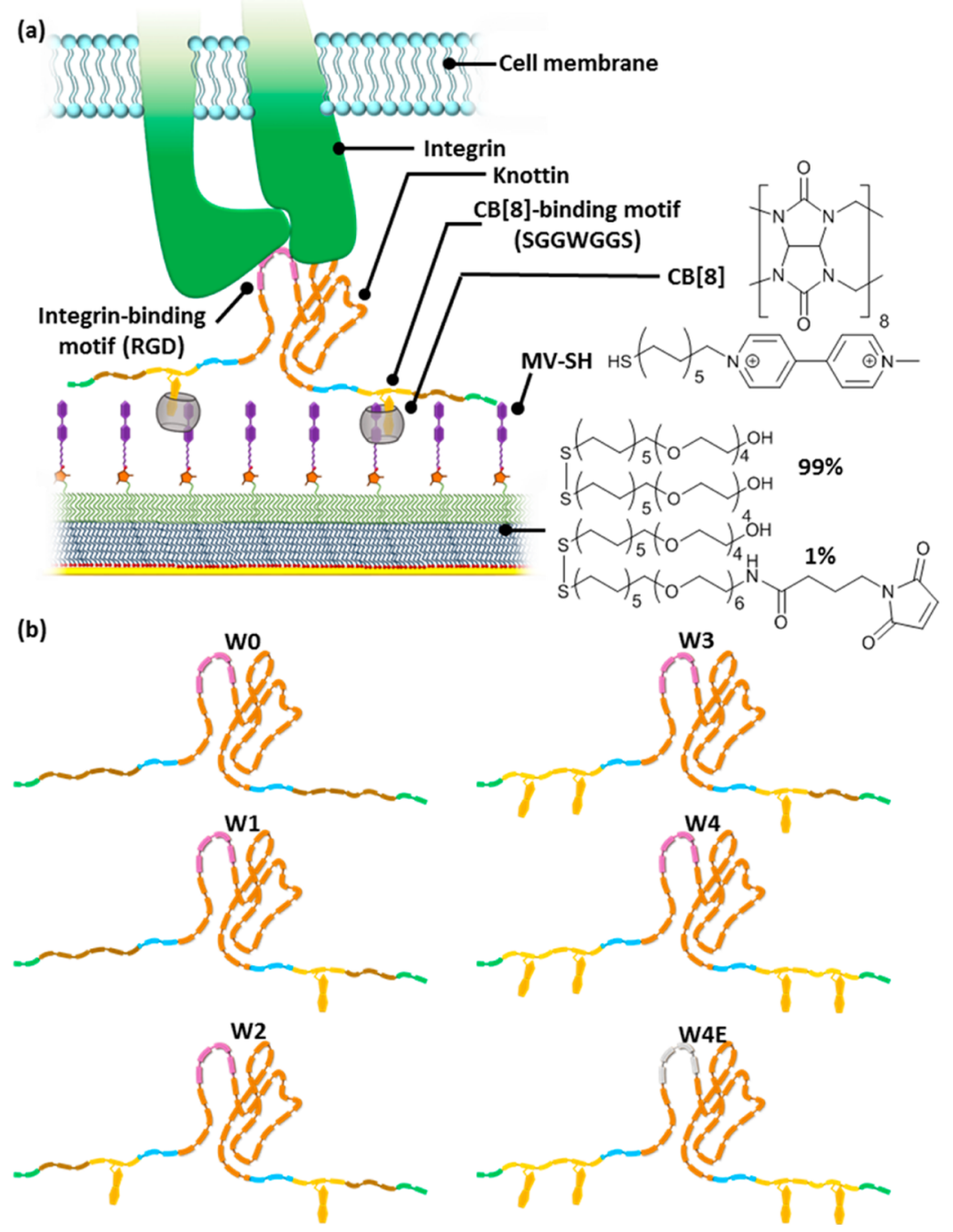

Figure 1. (a) Schematic representation of one of the integrin-binding knottin constructs (W2) noncovalently bound to a CB[8]-SAM through the formation of two ternary complexes. The drawings do not represent the natural size relationship. (b) Cartoon representing the set of knottin constructs prepared in this study. Different regions of the knottins indicate GEGK (green), SGGSGGS (brown), SGGWGGS (yellow, with appended tryptophan moiety), SGSGSG (blue), RGD (purple), RGE (gray), and the remainder of the knottin (orange).

displaying linear RGD using our reversible noncovalent system were nearly identical for up to several hours when compared to those of conventional irreversible covalent systems. ${ }^{20} \mathrm{~A}$ combination of factors account for this observation, among which the increase in the effective concentration of the supramolecular components under spread cells and the weak rupture force between linear RGD and integrin played major roles. $^{20,21}$ When using RGD with a high affinity for integrin receptors, the interaction between cells and our supramolecular surfaces would be more prone to sense differences in the binding affinity of the host-guest complex. Compared to linear RGD, improved integrin binding, functionality, and specificity have been achieved when using synthetic cyclic RGD sequences $^{22}$ and recombinant proteins with RGD grafted within one of their loops, for example, in VEGF, ${ }^{23}$ fluorescent proteins, $^{24}$ and miniprotein scaffolds. ${ }^{25,26}$ One such RGDcontaining loop with a high affinity $\left(K_{\mathrm{d}}<30 \mathrm{nM}\right)$ for integrins $\alpha_{\mathrm{V}} \beta_{3}, \alpha_{\mathrm{V}} \beta_{5}$, and $\alpha_{5} \beta_{1}^{27}$ was developed in a knottin, a highly stable cystine-stabilized miniprotein scaffold. Because of their knotted structure, these miniproteins have been shown to be highly resistant to proteolytic and thermal degradation. RGD- modified knottin was successfully used to inhibit tumor cell propagation. ${ }^{25,26}$ We have recently reported the assembly of knottins on our supramolecular SAMs in monovalent and bivalent manners. In this case, heteroternary complexes were formed each between surface-bound $\mathrm{CB}[8]-\mathrm{MV}^{2+}$ and tryptophan (Trp, W) moieties on either one or both of the $\mathrm{N}$ - and C-termini of the knottins. The affinity for the bivalent heteroternary complexation occurred with $K_{\mathrm{d}}=0.75 \mu \mathrm{M}$, more than an order of magnitude stronger than its monovalent variant. $^{28}$ Surface-tethered knottins remained functional and were found to specifically recognize their target binding enzyme, trypsin. ${ }^{28}$ Here we aim to exploit the multivalent nature of tryptophan and RGD-modified knottins to influence their interactions with cells

We approach this study by preparing a focused set of integrin-targeting knottin variants with varying affinities for our supramolecular $\mathrm{CB}[8]-\mathrm{SAM}$ surfaces. To this end, $\mathrm{CB}[8]$ SAMs were prepared on an antifouling layer on gold displaying $1 \%$ maleimide functionalities that were reacted with a thiolated methyl viologen $\left(\mathrm{MV}^{2+}\right)$. Subsequently, a ternary complex among $\mathrm{MV}^{2+}, \mathrm{CB}[8]$, and the knottin is formed. The 


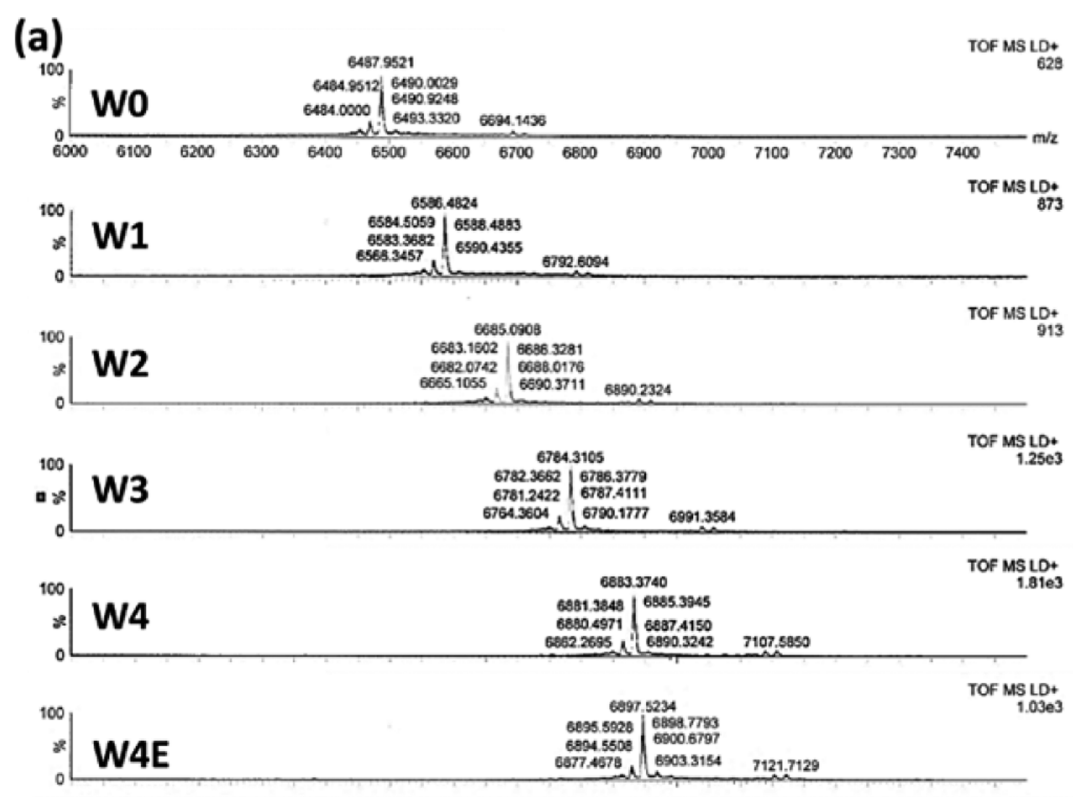

\begin{tabular}{|c|c|c|c|}
\hline $\begin{array}{l}\text { Knottin } \\
\text { construct }\end{array}$ & $\begin{array}{l}\text { Calc. } \\
(\mathrm{m} / \mathrm{z})\end{array}$ & $\begin{array}{l}\text { Calc. minus } \\
\text { GEGK }(\mathrm{m} / \mathrm{z})\end{array}$ & $\begin{array}{l}\text { Exp. } \\
(\mathrm{m} / \mathrm{z})\end{array}$ \\
\hline wo & 6865 & 6487 & 6488 \\
\hline W1 & 6964 & 6586 & 6586 \\
\hline W2 & 7063 & 6685 & 6685 \\
\hline W3 & 7162 & 6784 & 6784 \\
\hline W4 & 7261 & 6884 & 6883 \\
\hline W4E & 7275 & 6898 & 6898 \\
\hline
\end{tabular}

Figure 2. (a) Mass spectra of the cleaved and purified knottin constructs determined using MALDI-ToF and (b) masses $(\mathrm{m} / z)$ compared to calculated values.

differences in binding affinities for these $\mathrm{CB}[8]$-SAMs of a series of knottin constructs, given by the number of available tryptophan (W) residues on the knottins, as well as the effect of the knottins' valencies on cell adhesion were evaluated.

\section{RESULTS AND DISCUSSION}

Our genetically engineered knottin constructs that are able to simultaneously bind integrins and $\mathrm{CB}[8]$ were designed with the features as depicted in Figure 1. A knottin miniprotein (W) engineered by Cochran and co-workers ${ }^{27}$ to bind integrins $\alpha_{\mathrm{V}} \beta_{3}, \alpha_{\mathrm{V}} \beta_{5}$, and $\alpha_{5} \beta_{1}$ with high affinity $\left(K_{\mathrm{d}}<30 \mathrm{nM}\right)$ was selected for the current study and contains no other tryptophans. To either one of the $\mathrm{N}$ - or C-termini of the knottin, zero to two $\mathrm{CB}[8]$-binding motifs (SGGWGGS) were genetically fused, resulting in five different knottin constructs (W0, W1, W2, W3, and W4) as shown in Figure 1b. For the constructs with fewer than four SGGWGGS motifs, tryptophan was replaced by a serine, resulting in a nonbinding SGGSGGS motif that was placed at the remaining sites. These motifs were separated from the knottin by flexible linkers composed of glycines (Gly, G) and serines (Ser, S) with a maximum length of about $2.2 \mathrm{~nm}$, which is a sufficient distance to allow for host-guest interactions to occur between adjacent hosts on the surface and tryptophans residing on one end of the knottin. ${ }^{20}$
At the $\mathrm{N}$ - and C-termini of the entire constructs, zwitterionic GEGK peptide sequences were included to improve their solubility in aqueous solutions. As a negative control for the integrin-binding RGD motif, the functional loop of the W4 knottin was mutated to RGE, resulting in construct W4E (Figure 1b).

These knottin constructs were genetically fused to the Cterminus of a teal fluorescent protein (TFP), separated by an enterokinase cleavage site (EKCS) linker and expressed in E. coli to prevent inclusion in body formation as described previously. ${ }^{28,29}$ Once expressed, the knottins were cleaved from TFP using enterokinase and purified using centrifugal filters. (See the Supporting Information for details.) SDS-PAGE was performed using the mixture obtained after the cleavage reaction and before centrifugation where bands corresponding to TFP $(\sim 30 \mathrm{kDa})$, enterokinase $(\sim 25 \mathrm{kDa})$, and the knottin constructs $(\sim 10 \mathrm{kDa})$ were observed (Figure S1). The bands were visualized by enhancing the fluorescence of the tryptophan using Bio-Rad stain-free precast gels (Supporting Information). Accordingly, the W0 band was not visible, and the intensity of the bands corresponding to the other constructs seemed to depend upon the number of tryptophans they contained. 

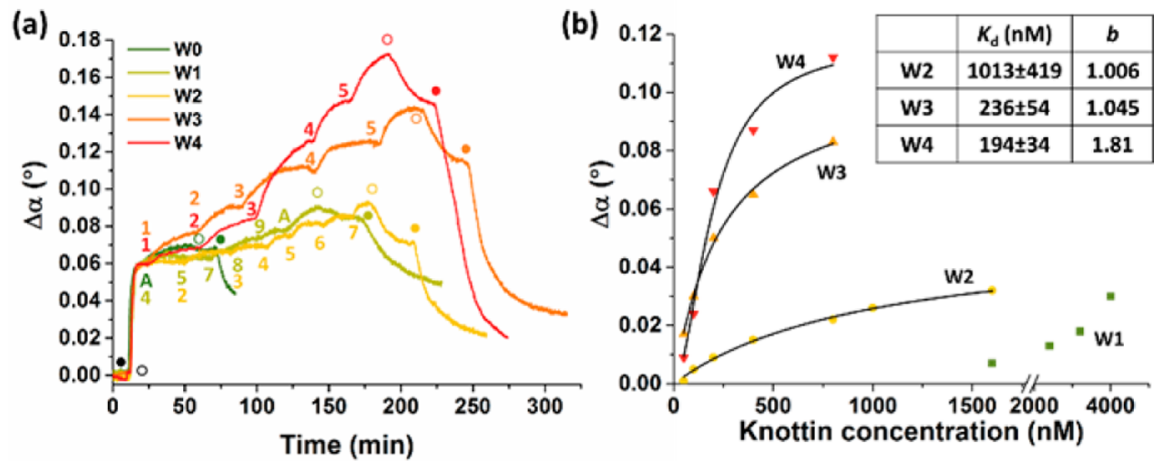

Figure 3. (a) SPR response plots observed when flowing different knottin constructs over $\mathrm{CB}[8]$ SAMs. $\bullet=$ buffer, $\mathrm{O}=50 \mu \mathrm{M} \mathrm{CB}[8], 1=50 \mathrm{nM}$, $2=100 \mathrm{nM}, 3=200 \mathrm{nM}, 4=400 \mathrm{nM}, 5=800 \mathrm{nM}, 6=1 \mu \mathrm{M}, 7=1.6 \mu \mathrm{M}, 8=2.4 \mu \mathrm{M}, 9=3.2 \mu \mathrm{M}$, and $\mathrm{A}=4.0 \mu \mathrm{M}$ knottin construct in the presence of $50 \mu \mathrm{M} \mathrm{CB}[8]$. All plots were normalized to the response of $\mathrm{CB}[8]$ binding once it reached equilibrium at $\Delta \alpha=0.06^{\circ}$. (b) SPR response values of the knottin constructs (in the presence of $50 \mu \mathrm{M} \mathrm{CB}[8]$ ) were plotted against corresponding concentrations of knottins. The solid lines represent fits using the four-parameter logistic regression model.

Through MALDI-ToF measurements, the masses of the purified knottin constructs were determined (Figure 2). Surprisingly, the recorded masses were always $378 \mathrm{Da}$ lower than the theoretically calculated masses. This mass difference corresponded exactly to that of the GEGK sequence at one of the termini of the knottin constructs. The enterokinase cleavage site (DDDDK) lies adjacent to the N-terminal GEGK sequence, and enterokinase is normally expected to specifically cleave the protein at the C-terminal of the lysine. In our construct, we suspect that the enterokinase detected its specific cleavage site; however, it performed the cleavage after the lysine residue in the $\mathrm{N}$-terminus GEGK sequence as well. ${ }^{30}$ Subsequently, the masses of the knottin constructs calculated by subtracting the mass of a GEGK sequence correspond to the experimentally determined masses. In the theoretically determined masses, we also took into account the 6 Da loss during the formation of all disulfide bridges within the knottin core. Because the experimental masses corresponded to these theoretical values, it can be assumed that all disulfide bridges were formed in our constructs. These results indicate that we were able to successfully isolate our knottin constructs using $E$. coli. To determine the concentrations of the cleaved and purified knottin constructs, UV-vis absorbance spectra were recorded (Figure S2). An absorbance peak observed at $\lambda=280$ $\mathrm{nm}$ was due to the presence of tryptophans, and another peak appeared at $\lambda=250 \mathrm{~nm}$ that was due to the presence of cystines. Using the absorbance values at $\lambda=280$ and $250 \mathrm{~nm}$ of these curves and their ratios, along with theoretically determined extinction coefficients, the concentration of each sample was determined. (The calculations are presented in the Supporting Information.)

The differences between the knottin constructs in binding to well-packed self-assembled monolayers (SAMs) of $\mathrm{MV}^{2+}$ $\mathrm{CB}[8]$ were evaluated. The monolayers were prepared (Figure 1a) on gold sensors for surface plasmon resonance (SPR) with a background layer of antifouling oligo(ethylene glycol) alkanethiols consisting of $1 \%$ maleimide groups. (See the details in the Supporting Information. ${ }^{31}$ ) Thiolated methyl viologen $\left(\mathrm{MV}^{2+}\right)$ was conjugated to the maleimide groups and acted as the first guest for $\mathrm{CB}[8]$ to bind at the surface. In Figure 3a, SPR measurements were performed in a flow cell using the $\mathrm{MV}^{2+}$-functionalized sensors over which $\mathrm{CB}[8]$ was flowed and allowed to interact. Following this, increasing concentrations of each knottin construct, in the presence of
$\mathrm{CB}[8]$, were sequentially injected and allowed to flow until equilibrium was reached. After the highest concentration, dissociation was allowed to occur by first flowing solutions with only $\mathrm{CB}[8]$, followed by buffer. It was observed that the binding responses of the knottin constructs were generally higher in the constructs containing more tryptophan with SGGWGGS motifs. In the case of W0, negligible binding was observed even when the highest concentration was used, supporting our claim that the knottin constructs bind to the $\mathrm{CB}[8]$ SAMs specifically through the tryptophan-containing motifs. It is important to note that tryptophan binds in the presence of $\mathrm{CB}[8]$ more strongly to surface-bound methyl viologen to form a heteroternary complex as compared to another tryptophan to form a homoternary complex in solution. ${ }^{32}$ In addition, depending on the number of tryptophan residues on the knottin constructs, a tryptophan on one knottin might dimerize intermolecularly with another tryptophan on a second knottin that remains in solution or potentially could lead to structures surface-bound by partial heterocomplexation and bulging from the surface. ${ }^{33}$ Alternatively, intramolecular binding is possible with another tryptophan linked by a flexible peptide chain on the same knottin. Intramolecular complexation is mostly probable on tetravalent W4 in which the tryptophan residues have more chances of interaction, with respect to trivalent W3. Much to our surprise, the higher the valency of the knottins, the higher the value where the SPR signal saturated, indicating that more knottins were bound to the surface the more binding sites that were available. At low concentrations of knottins, the SPR response for W3 was unexpectedly higher than for W4. Presumably, at low concentrations the interaction of the tetravalent construct with the surface in a heteroternary complex might suffer from the competing formation of divalent or tetravalent homoternary complexes in solution to a greater extent when compared to the W3 construct. Plotting the equilibrium-level responses against their respective concentrations for each knottin construct, apart from W0, gave binding curves (Figure $3 b$ ) from which we derived dissociation constants $\left(K_{\mathrm{d}}\right)$ and the slope at the inflection point (b) by analogy to dose-response curves in bioassays using a four-parameter logistic regression model. ${ }^{31}$ In the case of monovalent variant W1, no reliable fits were obtained because the saturation value at high concentrations was not accessible. For the higher-valent knottins, this analysis shows clearly that the affinity of the knottin constructs toward 

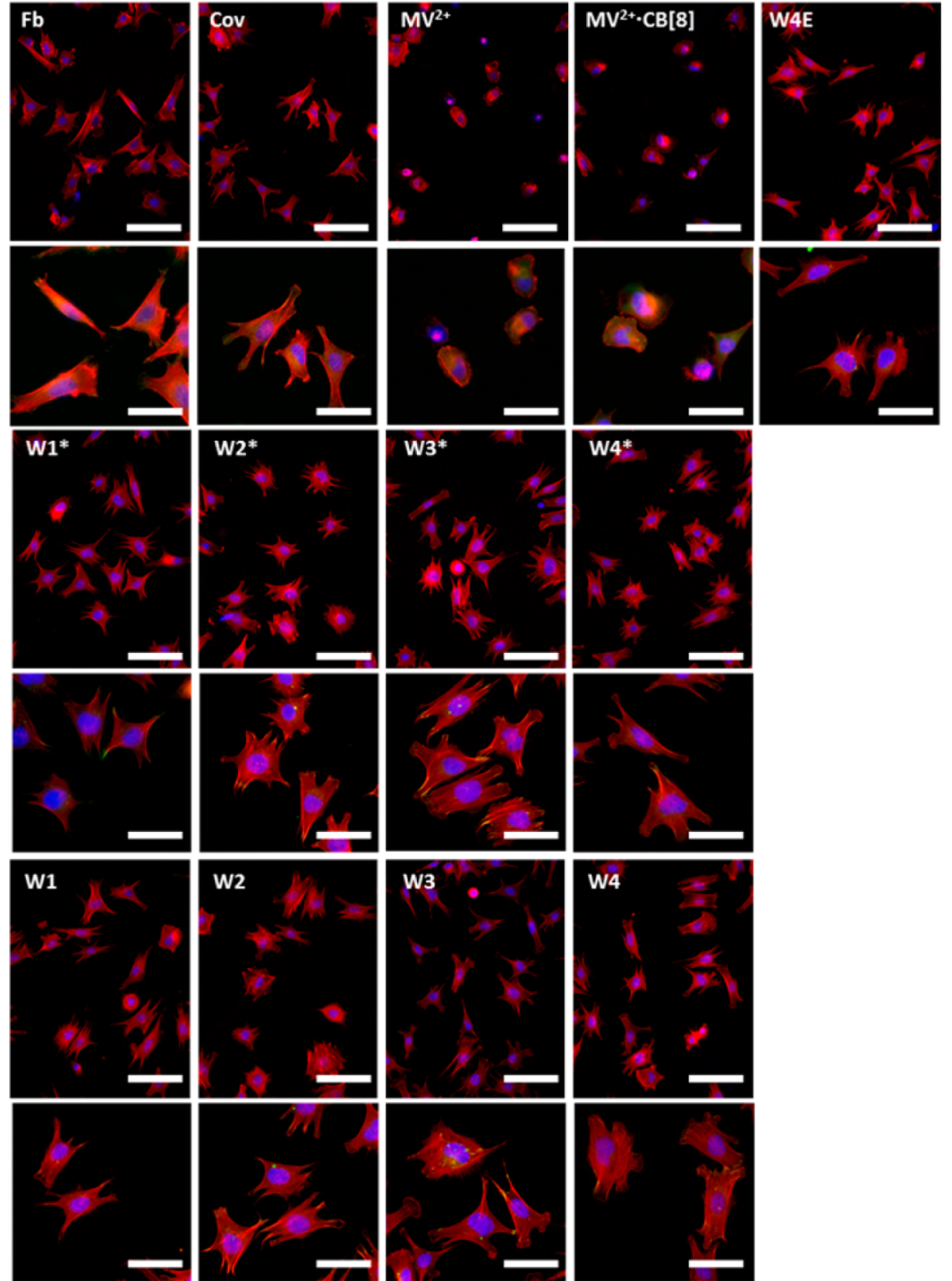

Figure 4. Fluorescence micrographs of $\mathrm{C} 2 \mathrm{C} 12$ cells on fibronectin $(\mathrm{Fb})$ surfaces or on gold SAMs modified with covalently attached RGD (Cov, GGCGGRGDS) or with $\mathrm{MV}^{2+}, \mathrm{MV}^{2+} \cdot \mathrm{CB}[8]$, or $\mathrm{MV}^{2+} \cdot \mathrm{CB}[8]$ and knottin variants. The star $\left({ }^{*}\right)$ indicates the density of knottin constructs, which is high when present and low when absent. Cells were stained for actin (red), nuclei (blue), and focal adhesion marker vinculin (green). Scale bars represent 100 and $50 \mu \mathrm{m}$ for the magnified images.

$\mathrm{CB}[8]$-SAMs increased as more SGGWGGS motifs were present in the knottin constructs, suggesting that binding occurred through multivalent interactions (Figure $3 \mathrm{~b}$ ). The bivalent knotting, W2, yielded a binding affinity value $\left(K_{\mathrm{d}}=1.0\right.$ $\pm 0.4 \mu \mathrm{M})$ that is comparable to what has been reported with similar host-guest systems by us previously, ${ }^{28}$ whereas W3 yielded a stronger binding affinity value of $K_{d}=0.24 \pm 0.05$ $\mu \mathrm{M}$. Interestingly, in the cases of W2 and W3, conventional Langmuir adsorption is present ( $b$ approaches 1$)$, whereas the binding of W4 occurred more strongly $\left(K_{\mathrm{d}}=0.19 \pm 0.03 \mu \mathrm{M}\right)$ but deviates from this ( $b$ exceeds 1$)$, indicating that the binding behavior changes with the knottin concentration. In addition, we note that the affinities do not strongly depend on valency, indicating extensive competition, and that higher values of saturation were observed for W3 and W4, even though all SAMs presented comparable $\mathrm{MV}^{2+}$ and $\mathrm{CB}[8]$ coverages (Figure $3 \mathrm{~b}$ ). Furthermore, the dissociation rate seems to be rather insensitive to the valency, most likely indicating that the constructs have valencies that are very similar to those of the surface (or at least for what does get released, Figure $3 b$ ).
These observations indicate that true tri/tetravalent binding to a surface with these knottin constructs is impossible, and we tentatively assign the observations to a concentration-dependent scenario in which homodimerization in solution is competing with the formation of the heteroternary complexation on the surface. The most probable explanation is the formation of multilayered structures in which heteroternary complexes with the $\mathrm{MV}^{2+} \cdot \mathrm{CB}[8]$-modified surfaces anchor buckles of polyvalent knottins held together by homoternary (tryptophan) $)_{2} \cdot \mathrm{CB}[8]$ complexes. Further support is found in the desorption traces of the constructs upon switching to knottin-free solutions: whereas the decrease in coverage upon switching to $\mathrm{CB}[8]$ solutions is small and similar across W2$\mathrm{W} 4$, the signal upon switching to buffer shows larger decreases for the constructs with larger numbers of tryptophans. This behavior confirms a larger extent of multilayer formation for the higher-valency constructs.

Having demonstrated the ability of our knottin constructs to bind specifically with $\mathrm{MV}^{2+}$. $\mathrm{CB}[8]-S A M s$, we next proceeded to test cell adhesion on these surfaces. To this end, SAMs were 

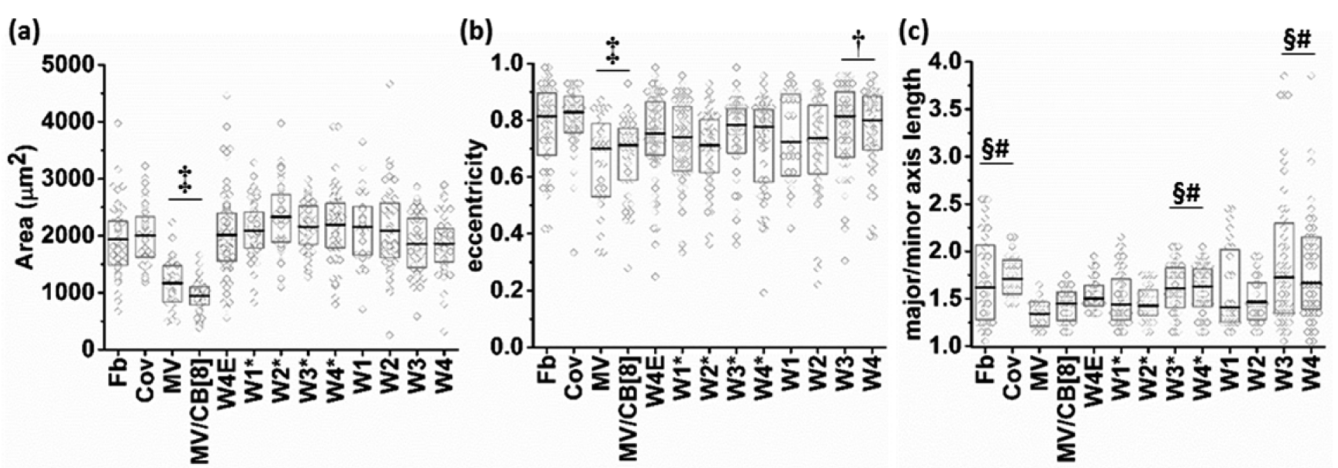

Figure 5. Box chart with binned data (30 bins) showing the (a) cell area, (b) eccentricity, and (c) ratio between the major and minor axis lengths of cells $(16 \leq n \leq 42)$, analyzed with CellProfiler software. The black lines represent the median, and gray lines represent the 25 th and 75 th percentiles. Mood's median test for non-normal distributions $(p \leq 0.05)$, in which populations are significantly different $(\ddagger)$ versus $\mathrm{Fb}, \mathrm{Cov}, \mathrm{W} 3 *, \mathrm{~W} 4 *, \mathrm{~W} 3$, and W4; $(\dagger)$ versus $\mathrm{W} 1$ and $\mathrm{W} 2 ;(\S)$ versus negative controls $\mathrm{MV}^{2+}, \mathrm{MV}^{2+} \mathrm{CB}[8]$, and W4E; and (\#) versus mono and bivalent knottin constructs $\mathrm{W} 1 *$, $\mathrm{W} 2 *$, W1, and $\mathrm{W} 2$.

prepared as for the SPR experiments (Supporting Information) with the mono-, bi-, tri- and tetravalent knottins (W1-W4) having on an exposed loop an RGD-binding motif with a lownanomolar binding affinity for integrins. This means that all of our knottin constructs bind more weakly to the surface than to the integrin and that differences in cell-binding behavior are primarily caused by differences in the host-guest interactions. Because variations in the cell environment in terms of ligand availability influence cell adhesion, ${ }^{34}$ two sets of surface densities were tested. The first set of conditions (high density) was prepared using $2 \mu \mathrm{M}$ knottin solutions, resulting in saturation for all polyvalent knottin constructs but different surface coverages depending on the valence. The second set (low density) was obtained by incubating the SAMs with knottin concentrations at which all constructs yielded approximately the same surface coverage $\left(\Delta \alpha=0.085^{\circ}\right.$ in Figure 3a). A common cell line for studying cell adhesion is the mouse myoblast $(\mathrm{C} 2 \mathrm{C} 12)$ cell line. $\mathrm{C} 2 \mathrm{C} 12$ cells were seeded and adhered to the SAMs for $1 \mathrm{~h}$, after which the cells were fixed and stained for actin, vinculin, and nuclei. The fluorescent micrographs are presented in Figure 4 at high (with an asterisk in Figure 4) and low (without an asterisk in Figure 4) surface coverage. To verify specific cell adhesion, the modified version of W4 was used in which the RGD motif was replaced by an RGE sequence (W4E). Additionally, surfaces modified with only $\mathrm{MV}^{2+}$ or with $\mathrm{MV}^{2+}$ and $\mathrm{CB}[8]$ were tested as negative controls. As positive controls for cell adhesion, fibronectin $(\mathrm{Fb})$-coated surfaces and SAMs presenting a covalently attached RGD peptide (Cov) were included (Figure 4). Cells adhered and spread on all protein surfaces at low and high densities of RGD-knottins, whereas fewer and rounded cells were seen in SAMs displaying only $\mathrm{MV}^{2+}$ or $\mathrm{MV}^{2+} \cdot \mathrm{CB}[8]$ (Figure 4). On SAMs of the control W4E knottin construct, fewer cells spread compared to the other knottin variants.

Quantitative analysis of the fluorescence micrographs was performed using CellProfiler software (Supporting Information). Attempts to quantify the extent of cell spreading considering the cell area gave no significant differences between the various SAMs, with the exception of negative controls $\mathrm{MV}^{2+}$ and $\mathrm{MV}^{2+} \cdot \mathrm{CB}[8]$ (Figure 5a). Further insights into the differences in cell morphology were achieved by comparing the cell circularity (eccentricity), which is a method approximating each cell by an ellipse and calculating the eccentricity as the ratio of the distance between the ellipse foci and the cell major axis length. An eccentricity value of 1 represents a perfect circle. The plot of the values of eccentricity is presented in Figure $5 \mathrm{~b}$. Negative controls $\mathrm{MV}^{2+}$ and $\mathrm{MV}^{2+}$. $\mathrm{CB}[8]$ were significantly different from the positive controls. We note that even though RGE is a commonly used inactive version of RGD we realized from this work that it does not necessarily have to be the case in our high-affinity integrinbinding knottins. In the original paper from which we adapted the current knottin sequence, they used a negative control with a completely different loop to render it completely integrininactive. $^{27}$ Despite this, we do see significant differences between the W4E and the W3 and W4 surfaces in terms of major/minor axis length and focal adhesions.

In addition, this major/minor axis length parameter allowed us to delineate a trend between cell elongation and valency when changing from the low-valency $\mathrm{W} 1$ and $\mathrm{W} 2$ knottins toward the high-valency W3 and W4 knottins. Significant differences between low- and high-valency knottins were found for the SAMs prepared at low density. To more clearly represent the cell elongation, the ratio between the major and minor cell axis lengths was evaluated and revealed to be more sensitive toward the differences in the valency of the knottins (Figure 5c). Significantly higher ratios, indicating larger elongation, were determined for cells seeded on surfaces presenting high-valency knottin constructs in comparison with cells on low valency ones, on both high and low surface densities. No differences were observed between the positive controls and the tri- and tetravalent constructs nor between all negative controls and the mono- and bivalent constructs at any surface density. This quantitative analysis confirmed that knottin constructs can be used to promote supramolecular cell adhesion and that constructs with different binding behavior can actually elicit differences in cell morphology. Interestingly, supramolecular SAMs at a low surface density of knottins yielded more marked differences in cell elongation with respect to surfaces with high surface density. The better performances of the surface at low knotting coverages could be due to an RGD ligand density closer to the optimum for $\mathrm{C} 2 \mathrm{C} 12$ cells as well as to the formation of more uniform monolayers when diluted solutions of knottins are provided for interaction with the surfaces. The distributions of the ratio between major and minor cell axis length presented in Figure $5 \mathrm{c}$ presumably indicate that cells on supramolecular SAMs with sufficient affinity resemble the cell elongation observed on $\mathrm{Fb}$ 

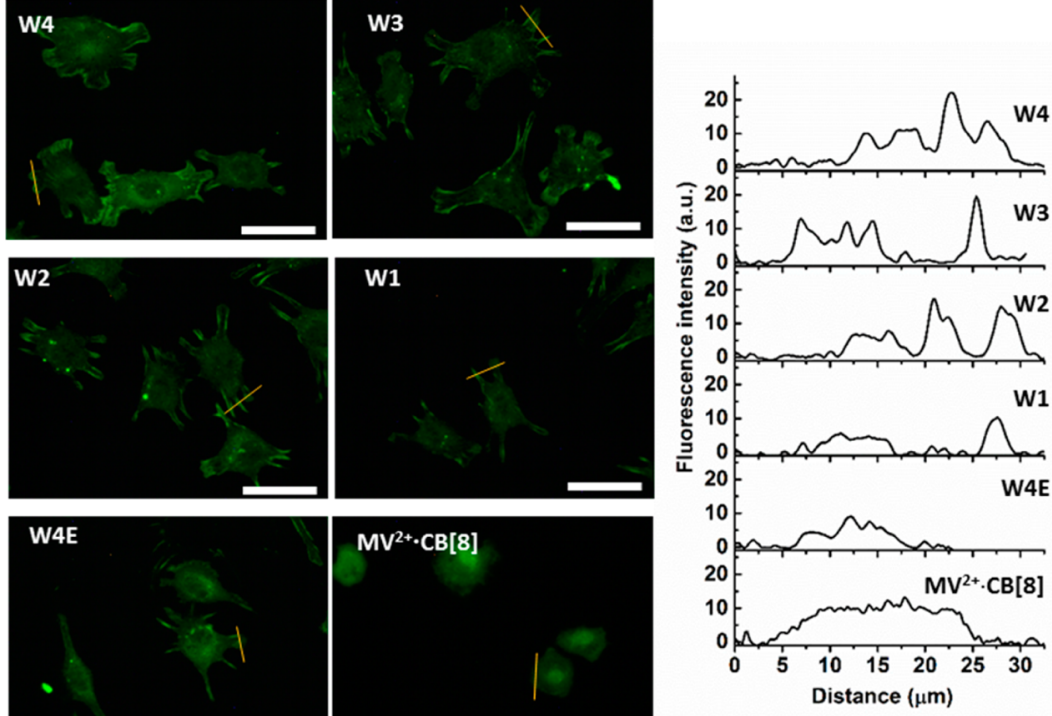

Figure 6. (Left panel) Fluorescence micrographs of $\mathrm{C} 2 \mathrm{C} 12$ cells on SAMs modified with $\mathrm{MV}^{2+} \cdot \mathrm{CB}[8]$ only or with knottin constructs W4E and W1-W4 at low surface density. Cells were stained for focal adhesion marker vinculin (green). Scale bars represent $50 \mu \mathrm{m}$. All images were taken with the same acquisition parameters. (Right panel) Intensity profiles for vinculin as indicated in the respective images in the left panel.

as natural mimics of the ECM. The green fluorescence of the vinculin marker for focal adhesions was also compared for cells seeded on knottin SAMs at low surface coverage (Figure 6). Along with the increase in valency, more pronounced localized green fluorescence intensity was detected. Oppositely, the green fluorescence intensity was uniformly spread in the cytoplasm in all negative controls. This confirmed the correlation between the valence of the knottins and the formation of mature focal adhesion clusters.

Cellular responses on multivalent supramolecular surfaces have been sparsely reported, even though such interactions are predominant in natural systems. SPR results clearly show that ligand assembly on the surface is affected by multivalency, leading to different types of surface coverages ranging from poor to multilayers. The preliminary cell studies provide an indication that the different types of ligand assemblies on the surface do affect certain aspects of cell behavior. The differences that were observed and quantified, even though they might seem modest, are indeed significant, as determined by appropriate statistical tests. Furthermore, significant differences seen between W3, W4 and W1, W3 for certain cell morphology parameters indicate that multivalency definitely plays a role in eliciting different cell behavior. Further cell experiments with more ligand assembly conditions, longer cell-spreading times, different cell types, and more in-depth analysis will be the subjects of upcoming studies.

\section{CONCLUSIONS}

We have investigated the $\mathrm{CB}[8]$-mediated assembly of multivalent knottins displaying RGD ligands for cell adhesion. A focused set of knottin constructs was produced and characterized with distinct numbers of tryptophans. The interaction of the knottins on $\mathrm{MV}^{2+} \cdot \mathrm{CB}[8]$ monolayers was characterized by SPR showing slightly higher binding affinities for knottins with a larger number of tryptophan residues. More pronounced was an increased extent of multilayer formation for the higher-valent constructs attributed to homoternary complex formation between tryptophans of different knottins and
$\mathrm{CB}[8]$. Cell adhesion on the supramolecular SAMs demonstrated the availability of the RGD ligand for specific integrin recognition on the knottins displayed on the supramolecular SAMs. Moreover, a correlation was observed between the elongation of cells and the valence of the knottins on the supramolecular SAMs. Tri- and tetravalent knottin constructs, especially at low surface density, yielded the largest extent of cell elongation and more pronounced focal adhesion formation, similar to that observed on fibronectin surfaces. This study the first step toward using knottins in dynamic and reversible biointerfaces and biomaterials.

\section{ASSOCIATED CONTENT}

\section{S Supporting Information}

The Supporting Information is available free of charge on the ACS Publications website at DOI: 10.1021/acs.langmuir.7b00702.

General methods, molecular cloning of knottin constructs, protein expression and purification, knottin concentration determination, MALDI-ToF, synthesis of thiol-functionalized $\mathrm{MV}^{2+}$, surface functionalization, surface plasmon resonance, cell culture, and data analysis (PDF)

\section{AUTHOR INFORMATION}

\section{Corresponding Author}

*E-mail: p.jonkheijm@utwente.nl.

\section{ORCID}

Jurriaan Huskens: 0000-0002-4596-9179

Pascal Jonkheijm: 0000-0001-6271-0049

\section{Present Address}

${ }^{\S}$ Dynamic Biomaterials, INM - Leibniz-Institut für Neue Materialien $\mathrm{GmbH}$, Campus D2 2, 66123 Saarbrücken, Germany.

\section{Author Contributions}

"Co-first authors. 


\section{Funding}

The European Research Council is acknowledged for funding through Starters Grant Sumoman 259183.

Notes

The authors declare no competing financial interest.

\section{ACKNOWLEDGMENTS}

Dr. Jenny Brinkmann is acknowledged for discussing cell experiments and for the gift of the cysteine-RGD.

\section{REFERENCES}

(1) Mouw, J. K.; Ou, G.; Weaver, V. M. Extracellular matrix assembly: a multiscale deconstruction. Nat. Rev. Mol. Cell Biol. 2014, 15 (12), 771-785.

(2) Satav, T.; Huskens, J.; Jonkheijm, P. Effects of variations in ligand density on cell signaling. Small 2015, 11 (39), 5184-5199.

(3) Hynes, R. O. Integrins: versatility, modulation, and signaling in cell adhesion. Cell 1992, 69 (1), 11-25.

(4) Ridley, A. J.; Schwartz, M. A.; Burridge, K.; Firtel, R. A.; Ginsberg, M. H.; Borisy, G.; Parsons, J. T.; Horwitz, A. R. Cell migration: integrating signals from front to back. Science 2003, 302 (5651), 1704-1709.

(5) Ruoslahti, E. RGD and other recognition sequences for integrins. Annu. Rev. Cell Dev. Biol. 1996, 12, 697-715.

(6) Discher, D. E.; Mooney, D. J.; Zandstra, P. W. Growth factors, matrices, and forces combine and control stem cells. Science 2009, 324 (5935), 1673-1677.

(7) Mrksich, M. Using self-assembled monolayers to model the extracellular matrix. Acta Biomater. 2009, 5 (3), 832-841.

(8) Choi, I.; Yeo, W. S. Self-assembled monolayers with dynamicity stemming from (bio)chemical conversions: from construction to application. ChemPhysChem 2013, 14 (1), 55-69.

(9) Brinkmann, J.; Cavatorta, E.; Sankaran, S.; Schmidt, B.; Van Weerd, J.; Jonkheijm, P. About supramolecular systems for dynamically probing cells. Chem. Soc. Rev. 2014, 43 (13), 4449-4469.

(10) Rosales, A. M.; Anseth, K. S. The design of reversible hydrogels to capture extracellular matrix dynamics. Nat. Rev. Mats. 2016, 1, 15012 .

(11) Webber, M. J.; Appel, E. A.; Meijer, E. W.; Langer, R. Supramolecular biomaterials. Nat. Mater. 2015, 15 (1), 13-26.

(12) An, Q.; Brinkmann, J.; Huskens, J.; Krabbenborg, S.; De Boer, J.; Jonkheijm, P. A supramolecular system for the electrochemically controlled release of cells. Angew. Chem., Int. Ed. 2012, 51 (49), 12233-12237.

(13) Park, K. M.; Yang, J. A.; Jung, H.; Yeom, J.; Park, J. S.; Park, K. H.; Hoffman, A. S.; Hahn, S. K.; Kim, K. In situ supramolecular assembly and modular modification of hyaluronic acid hydrogels for 3D cellular engineering. ACS Nano 2012, 6 (4), 2960-2968.

(14) Boekhoven, J.; Rubertpérez, C. M.; Sur, S.; Worthy, A.; Stupp, S. I. Dynamic display of bioactivity through host-guest chemistry. Angew. Chem., Int. Ed. 2013, 52 (46), 12077-12080.

(15) Neirynck, P.; Brinkmann, J.; An, Q.; Van Der Schaft, D. W. J.; Milroy, L. G.; Jonkheijm, P.; Brunsveld, L. Supramolecular control of cell adhesion via ferrocene-cucurbit[7] uril host-guest binding on gold surfaces. Chem. Commun. 2013, 49 (35), 3679-3681.

(16) Petkau-Milroy, K.; Brunsveld, L. Supramolecular chemical biology; bioactive synthetic self-assemblies. Org. Biomol. Chem. 2013, $11(2), 219-232$.

(17) Li, H.; Frith, J.; Cooper-White, J. J. Modulation of stem cell adhesion and morphology via facile control over surface presentation of cell adhesion molecules. Biomacromolecules 2014, 15 (1), 43-52.

(18) Neirynck, P.; Schimer, J.; Jonkheijm, P.; Milroy, L. G.; Cigler, P.; Brunsveld, L. Carborane- $\beta$-cyclodextrin complexes as a supramolecular connector for bioactive surfaces. J. Mater. Chem. B 2015, 3 (4), 539545.

(19) Rodell, C. B.; Mealy, J. E.; Burdick, J. A. Supramolecular guesthost interactions for the preparation of biomedical materials. Bioconjugate Chem. 2015, 26 (12), 2279-2289.
(20) Sankaran, S.; Jaatinen, L.; Brinkmann, J.; Zambelli, T.; Vörös, J.; Jonkheijm, P. Cell adhesion on dynamic supramolecular surfaces probed by fluid force microscopy-based single-cell force spectroscopy. ACS Nano 2017, 11, 3867.

(21) Khan, T. A.; Wang, X.; Maynard, J. A. Inclusion of an RGD motif alters invasin integrin-binding affinity and specificity. Biochemistry 2016, 55 (14), 2078-2090.

(22) Haubner, R.; Finsinger, D.; Kessler, H. Stereoisomeric peptide libraries and peptidomimetics for designing selective inhibitors of the $\alpha(\mathrm{v}) \beta 3$ integrin for a new cancer therapy. Angew. Chem., Int. Ed. Engl. 1997, 36 (13-14), 1374-1389.

(23) Papo, N.; Silverman, A. P.; Lahti, J. L.; Cochran, J. R. Antagonistic VEGF variants engineered to simultaneously bind to and inhibit VEGFR2 and $\alpha_{\mathrm{v}} \beta_{3}$ integrin. Proc. Natl. Acad. Sci. U. S. A. 2011, 108 (34), 14067-14072.

(24) Sonntag, M. H.; Schill, J.; Brunsveld, L. Integrin-targeting fluorescent proteins: exploration of RGD insertion sites. ChemBioChem 2017, 18 (5), 441-443.

(25) Kim, J. W.; Cochran, F. V.; Cochran, J. R. A chemically crosslinked knottin dimer binds integrins with picomolar affinity and inhibits tumor cell migration and proliferation. J. Am. Chem. Soc. 2015, 137 (1), 6-9.

(26) Cox, N.; Kintzing, J. R.; Smith, M.; Grant, G. A.; Cochran, J. R. Integrin-targeting knottin peptide-drug conjugates are potent inhibitors of tumor cell proliferation. Angew. Chem., Int. Ed. 2016, 55 (34), 9894-9897.

(27) Kimura, R. H.; Levin, A. M.; Cochran, F. V.; Cochran, J. R. Engineered cystine knot peptides that bind $\alpha \mathrm{v} \beta 3, \alpha \mathrm{v} \beta 5$, and $\alpha 5 \beta 1$ integrins with low-nanomolar affinity. Proteins: Struct., Funct., Genet. 2009, 77 (2), 359-369.

(28) Sankaran, S.; De Ruiter, M.; Cornelissen, J. J. L. M.; Jonkheijm, P. Supramolecular surface immobilization of knottin derivatives for dynamic display of high affinity binders. Bioconjugate Chem. 2015, 26 (9), 1972-1980.

(29) Sankaran, S.; Stojanovic, I.; Barendregt, A.; Heck, A. J. R.; Schasfoort, R. B. M.; Jonkheijm, P. Scaffolding of cystine-stabilized miniproteins. ChemistrySelect 2016, 1 (5), 1039-1046.

(30) Shahravan, S. H.; Qu, X.; Chan, I. S.; Shin, J. A. Enhancing the specificity of the enterokinase cleavage reaction to promote efficient cleavage of a fusion tag. Protein Expression Purif. 2008, 59 (2), 314319.

(31) Sankaran, S.; Kiren, M. C.; Jonkheijm, P. Incorporating bacteria as a living component in supramolecular self-assembled monolayers through dynamic nanoscale interactions. ACS Nano 2015, 9 (4), 3579-3586.

(32) Bush, M. E.; Bouley, N. D.; Urbach, A. R. Charge-mediated recognition of $\mathrm{N}$-terminal tryptophan in aqueous solution by a synthetic host. J. Am. Chem. Soc. 2005, 127 (41), 14511-14517.

(33) Hou, C.; Li, J.; Zhao, L.; Zhang, W.; Luo, Q.; Dong, Z.; Xu, J.; $\mathrm{Liu}, \mathrm{J}$. Construction of protein nanowires through cucurbit[8]urilbased highly specific host-guest interactions: an approach to the assembly of functional proteins. Angew. Chem., Int. Ed. 2013, 52 (21), 5590-5593.

(34) Liu, Y.; Medda, R.; Liu, Z.; Galior, K.; Yehl, K.; Spatz, J. P.; Cavalcanti-Adam, E. A.; Salaita, K. Nanoparticle tension probes patterned at the nanoscale: impact of integrin clustering on force transmission. Nano Lett. 2014, 14 (10), 5539-5546. 